\title{
What still presents urgently to ENT during a pandemic - Experience of an ENT rapid access clinic during COVID-19
}

\author{
Thomas Stubington $^{1}$, Bethaney Morrison ${ }^{1}$, Christian Sevilla ${ }^{1}$, and Owen Judd ${ }^{1}$ \\ ${ }^{1}$ Royal Derby Hospital
}

July 13, 2020

\begin{abstract}
* Telephone consultations are effective in mild epistaxis and nasal trauma * Telephone follow-ups can save hospital attendances particularly in peritonsillar abscess and certain cases of otitis externa * Otitis externa and foreign bodies tend to require faceto-face consultations * Otitis externa was the most common reason for semi-urgent ENT consultation whilst social restrictions were in place
\end{abstract}

\section{Key Points}

- Telephone consultations are effective in mild epistaxis and nasal trauma

- Telephone follow-ups can save hospital attendances particularly in peritonsillar abscess and certain cases of otitis externa

- Otitis externa and foreign bodies tend to require face-to-face consultations

- Otitis externa was the most common reason for semi-urgent ENT consultation whilst social restrictions were in place

\section{Keywords}

COVID-19, Urgent, Telephone

\section{Introduction}

COVID-19 has been declared an international pandemic. It is thought that COVID-19 is spread via respiratory droplets necessitating the use of personal protective equipment (PPE) when assessing and managing suspected cases ${ }^{1}$. The nature of ear nose and throat (ENT) examinations may put practitioners at high risk of transmission ${ }^{2}$ and this has led ENTUK to release specific guidelines ${ }^{3}$. Where possible face to face consultations are minimised and telephone consultations used. Where this cannot be achieved appropriate PPE is essential. ${ }^{4}$

Prior to the COVID 19 pandemic, a rapid access triage clinic was run by the junior ENT doctor holding the referral bleep. With the advent of COVID-19, the ENT rapid access clinic was streamlined to provide review by a senior clinician to facilitate treatment and discharge in as many cases as possible. The intention was to reduce admission rates and potential exposure to COVID positive inpatients in the main hospital. This initial report presents data from the first 5 weeks, demonstrating the range of conditions that are still 
presenting to ENT on a semi-urgent basis. We also discuss the patients who require repeat consultations, despite the transmission risk associated with multiple attendances.

\section{Methods}

The Emergency clinic online booking calendar was accessed and all bookings reviewed over a 5 week period from $23^{\text {rd }}$ March to $24^{\text {th }}$ April. Presenting complaint and patient history were obtained from the booking system and further demographic information was obtained from the hospital's electronic record system. Where possible, outcomes were obtained either from letters or the electronic records system. However, due to COVID-19 and pressures on the audit department, patient records contained exclusively in the paper case notes could not be retrieved. Data analysis was carried out in Microsoft excel and figures were also produced in Excel.

\section{Results}

This audit included a total of 97 appointments during a 5-week period.

\section{Indications for Consultation}

Figure 1 shows the indications for each individual consultation,including re-attendances by the same patient. Otitis externa was by far the most frequent indication for an appointment, followed by epistaxis and foreign bodies in the ear. Surprisingly, patients whose appointments had been cancelled or postponed due to the pandemic made up only $1 \%$.

\section{Repeat Consultations}

Repeat attendances made up 26/97 episodes of patient care. The most frequent diagnoses when adjusted to not include re-attenders are; foreign bodies (18\%), epistaxis (17\%) and OE (15\%).. Of these re-attendances 13 were planned repeat consultations arranged by ENT, 11 were either via ED or repeat GP referral and in 2 cases the patient called the department. The greatest number of repeat appointments was 5 in an otitis externa patient, this patient had unfortunately been mismanaged by the GP and was resistant to further

advice. Aside from this all remaining patients had either 2 or 3 appointments and 9 of the 16 patients had at least one phone appointment. Overwhelmingly the most common diagnosis in patients with repeat appointments was otitis externa followed by epistaxis nasal trauma and bleeding ears (Figure 2)

\section{Age Distribution}

The most frequently attending age group (Figure 3) was 0-9, primarily due to foreign bodies. Accounting for repeat appointments did not have a significant effect on age distribution.

\section{Telephone Consultations}

As described in Table 1, 26/97 appointments were carried out over the telephone. A total of 13 patients were solely managed with telephone consultations. 


\section{Discussion}

On the $23^{\text {rd }}$ of March 2020, the United Kingdom announced social isolation steps to reduce the spread of coronavirus. These steps, hitherto referred to as "lockdown", brought about dramatic social changes. This dramatic change in the lifestyle of the British public may help to explain some of the trends emerging within the patients seen in our emergency clinic.

\section{Indication for consultation}

Otitis Externa was a frequent indication despite typically being mild and self-limiting. The majority of these patients had undergone prior telephone appointments with their general practitioner (GP) who had prescribed oral antibiotics. Although inappropriate use of oral antibiotics by GPs is a common problem in the COVID era, this may be due to concerns around the risks of otoscopy. Without examining the ear, GPs are likely to be reluctant to prescribe topical preparations for fear of ototoxicity. Despite the high proportion in the clinic, this is likely still under what you would expect. Using a $1.3 \%$ annual incidence and a $3 \%$ referral rate to $\mathrm{ENT}^{5}$, one might expect around 23 cases over a 5 week period (based on UK population of $66,435,600$ and the Royal Derby serving a population of 600,000). Accepting the extrapolations inherent in this calculation, one possible explanation for this is that reduced water exposure as a result of swimming pool and beach closures has resulted in fewer cases.

Presentations with foreign bodies and nasal trauma are also potentially explained by social restrictions. Foreign body presentations were mainly in children, likely frustration as a result of being stuck indoors. We anticipated that reduced car travel and opportunities for sports would reduce nasal traumas. Sadly, lockdown measures around the world have led to a rise in domestic violence cases ${ }^{6}$. Additionally, with elderly patients being advised to self-isolate, they may not have the level of support to mobilise that they usually would predisposing them to falls and injuries.

Peritonsillar abscess and tonsillitis represented only $6 \%$. Social distancing has likely impacted the spread of upper respiratory tract infections. The 2002 UK national audit quoted 30 peritonsillar abscesses as the average number treated per year ${ }^{7}$ which would make 3 cases over a 5 week period appropriate. However, previously published data from the Royal Derby Hospital demonstrates a rate of 43 over a period of 9 months ${ }^{8}$. Extrapolating this, 5-6 cases over a period of 5 weeks could be expected.

One group of patients with a surprisingly low frequency are the patients who have had outpatient appointments cancelled or rescheduled. We expected that large numbers of patients with postponed appointments would present via their GPs, but so far this has not been the case. This will likely increase as patients' symptoms become more troublesome.

\section{Age distribution}

The high proportion of children was almost exclusively due to foreign bodies. In the UK, those over 70 have been advised to self-isolate completely. It is interesting to see that compliance with these measures is reflected in the low proportion of patients in this age group presenting to the emergency clinic. The age group 50-59 is more difficult to explain. Individuals in this age group, though likely still working, may well be assisting older relatives but are in an age group where they are likely to have some co-morbidities. It is possible that because they are less isolated than older populations, they are more willing to present to their GPs and hence more likely to be referred. 


\section{Utility of telephone appointments}

A key recommendation from the ENTUK guidelines for COVID was to implement telephone based consultations where practical ${ }^{3,9}$. concerned potential concern wasthat the need for examination to reach a diagnosis for many ENT pathologies may limit the usefulness of telephone consultations. This dataset suggests that for certain pathologies telephone consultations can be highly effective. In some cases of epistaxis and nasal trauma, a telephone call was the only appointment required. We have changed our practice such that patients with nasal trauma are primarily managed over the telephone. They are telephoned at 7-days post injury and are only brought in for manipulation if they have ongoing concerns regarding appearance or obstruction. In epistaxis, minor bleeds with no risk factors or a clear precipitating trauma are managed over the telephone. Telephone consultations were also effective to check treatment efficacy in patients managed in ED. Peritonsillar abscesses and certain cases of otitis externa were ideal cases for this. Ambulatory management of peritonsillar abscess is well established in the literature ${ }^{8,10}$ and telephone follow-up is sufficient, provided the abscess has been adequately drained. Similarly, in clear cut cases of otitis externa where appropriate treatment has not yet been given pain and discharge were used as measures of improvement over the telephone

\section{Conclusion}

The data from our emergency clinic has demonstrated that telephone appointments and follow-up can be effective for acute presentations to ENT. Certain pathologies such as nasal trauma can be managed effectively via telephone consultation, whereas others, such as otitis externa and foreign bodies, require physical attendance and review by an ENT specialist. In-person appointments may also be necessary for ongoing problems.A surprising finding was the number of patients presenting with otitis externa, however this condition is often mismanaged and sometimes requires aural toileting, which can only be carried out in person by an ENT specialist.

\section{References}

1. Khan MM, Parab SR. Simple Economical Solution for Personal Protection Equipment (Face Mask/Shield) for Health Care Staff During COVID 19. Indian J Otolaryngol Head Neck Surg [Internet]. 2020 [cited 2020 May 9];1-5. Available from: http://link.springer.com/10.1007/s12070-020-01863-4

2. Krajewska J, Krajewski W, Zub K, Zatoński T. COVID-19 in otolaryngologist practice: a review of current knowledge. European Archives of Oto-Rhino-Laryngology. Springer; 2020.

3. ENT UK. Guidance for ENT during the COVID-19 pandemic [Internet]. 2020 [cited 2020 May 9]. Available from: https://www.entuk.org/guidance-ent-during-covid-19-pandemic

4. Hettiarachtchy S, Deakin D. Guidance for surgeons working during the COVID-19 pandemic - The Surgical Royal Colleges of the United Kingdom and Ireland [Internet]. 2020 [cited 2020 May 9]. Available from: https://www.rcseng.ac.uk/coronavirus/joint-guidance-for-surgeons-v1/

5. Rowlands S, Devalia H, Smith C, Hubbard R, Dean A. Otitis externa in UK general practice: A survey using the UK General Practice Research Database. Br J Gen Pract. 2001 Jul 1;51(468):533-8.

6. Bradbury-Jones C, Isham L. The pandemic paradox: The consequences of COVID-19 on domestic violence. J Clin Nurs [Internet]. 2020 Apr 22 [cited 2020 May 9];jocn.15296. Available from: https://onlinelibrary.wiley.com/doi/abs/10.1111/jocn.15296

7. Mehanna HM, Al-Bahnasawi L, White A. National audit of the management of peritonsillar abscess. Postgrad Med J. 2002 Sep 1;78(923):545-8. 
8. Judd O, Wilkinson S. Evaluation of ambulatory peritonsillar abscess management in our institution-our experience with 47 patients. 2016 [cited 2020 May 15]; Available from: https://scitest.custintgroup.com/handle/123456789/759

9. ENT UK. Exiting the pandemic: guidance for resuming ENT services [Internet]. London; 2020 [cited 2020 May 14]. Available from: https://www.entuk.org/exiting-pandemic-guidance-resuming-ent-services

10. Al Yaghchi C, Cruise A, Kapoor K, Singh A, Harcourt J. Out-patient management of patients with a peritonsillar abscess. Clin Otolaryngol. 2008 Feb;33(1):52-5.

Figure 1: Frequency of each diagnosis seen in clinic by individual appointment. This table includes repeat attendances for the same pathology as separate data points.

Figure 2: Frequency of each diagnosis in appointments made for a repeat attendance with the same problem.

Figure 3: Age distribution in patients presenting to the rapid access clinic.

Table 1: Breakdown of the patients who were managed by telephone consultation. Including indication for appointment. Unless otherwise specified the number represents the number of different patients with the pathology (i.e 3 epistaxis means 3 telephone appointments for 3 different patients with epistaxis)

\section{Hosted file}

Table 1.docx available at https://authorea.com/users/318332/articles/468684-what-stillpresents-urgently-to-ent-during-a-pandemic-experience-of-an-ent-rapid-access-clinicduring-covid-19

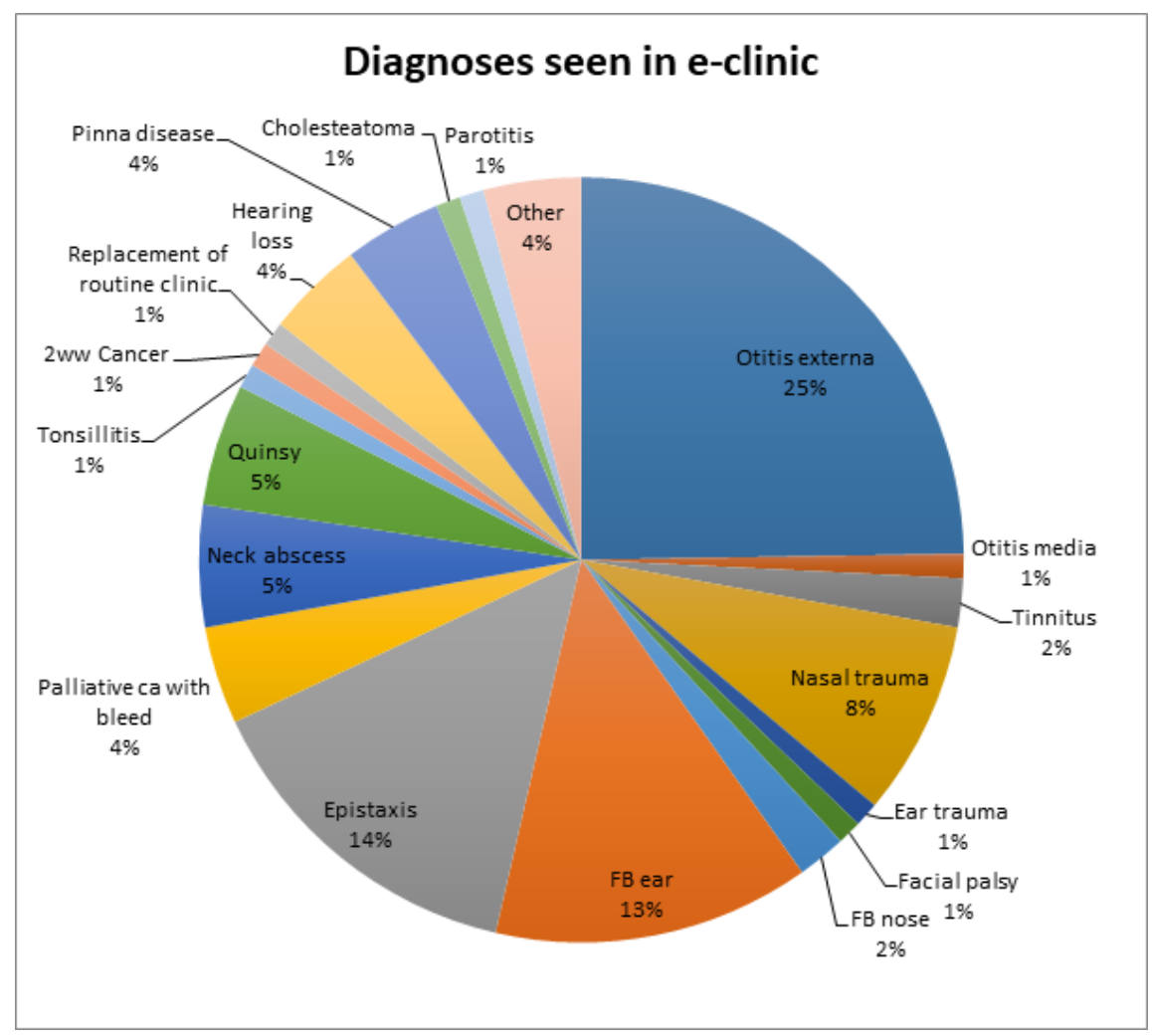




\section{Diagnosis as percentage of reattenders}

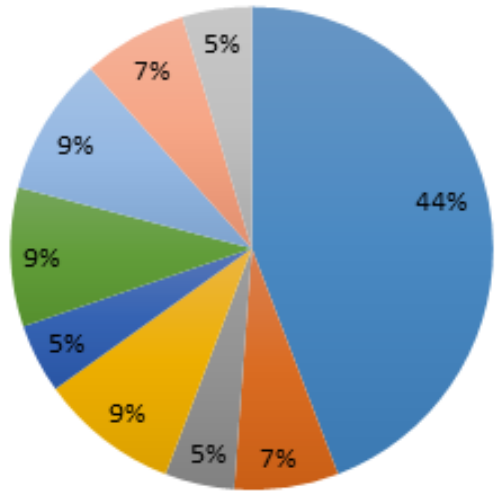

- Otit is Externa

- Palliative Ca w Bleed

Bleeding ear

Epistaxis

- Hearing loss

Quinsy

nasal trauma

Pinna ce llulitis

Neck Abscess

\section{Age of patients seen in clinic}

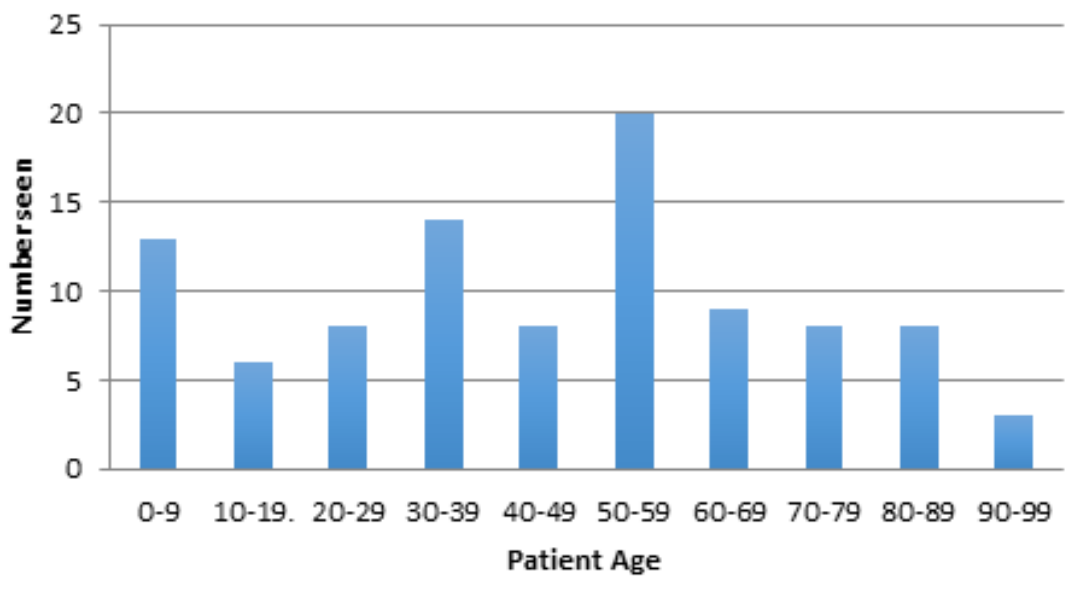

